\title{
A Kinematics Analysis Of Three Best 100 M Performances Ever
}

\author{
by \\ Maćkała Krzysztof ${ }^{1}$ Antti Mero ${ }^{2}$
}

\begin{abstract}
The purpose of this investigation was to compare and determine the relevance of the morphological characteristics and variability of running speed parameters (stride length and stride frequency) between Usain Bolt's three best $100 \mathrm{~m}$ performances. Based on this, an attempt was made to define which factors determine the performance of Usain Bolt's sprint and, therefore, distinguish him from other sprinters. We analyzed the previous world record of 9.69 s set in the 2008 Beijing Olympics, the current record of 9.58 s set in the 2009 Berlin World Championships in Athletics and the O lympic record of 9.63 s set in 2012 London Olympics Games by Usain Bolt. The application of VirtualDub Programme allowed the acquisition of basic kinematical variables such as step length and step frequency parameters of $100 \mathrm{~m}$ sprint from video footage provided by NBC TV station, BBC TV station. This data was compared with other data available on the web and data published by the Scientific Research Project Office responsible on behalf of IAAF and the German Athletics Association (DVL). The main hypothesis was that the step length is the main factor that determines running speed in the 10 and $20 \mathrm{~m}$ sections of the entire $100 \mathrm{~m}$ distance. Bolt's anthropometric advantage (body height, leg length and liner body) is not questionable and it is one of the factors that makes him faster than the rest of the finalists from each three competitions. Additionally, Bolt's $20 \mathrm{~cm}$ longer stride shows benefit in the latter part of the race. Despite these factors, he is probably able to strike the ground more forcefully than rest of sprinters, relative to their body mass, therefore, he might maximize his time on the ground and to exert the same force over this period of time. This ability, combined with longer stride allows him to create very high running speed - over $12 \mathrm{~m} / \mathrm{s}(12.05-12.34 \mathrm{~m} / \mathrm{s})$ in some $10 \mathrm{~m}$ sections of his three $100 \mathrm{~m}$ performances. These assumption confirmed the application of Ballerieich's formula for speed development. In most $10 \mathrm{~m}$ sections of the $100 \mathrm{~m}$ sprint, the step length was the parameter that significantly determined the increase of maximal running speed, therefore, distinguishing Bolt from the other finalists.
\end{abstract}

Key words: sprinting, world record, kinematic analysis, anthropometric characteristics

\section{Introduction}

In the last four years Usain Bolt improved the world record in the $100 \mathrm{~m}$ sprint three times, from $9.74 \mathrm{~s}$ to $9.58 \mathrm{~s}$. Over the last 40 years this record has been revised up to thirteen times from $9.95 \mathrm{~s}$ to $9.58 \mathrm{~s}$. The improvement equals $0.37 \mathrm{~s}$ (from 1968 to 2009) which is an increase in performance of $3.72 \%$. By comparison, during the same time period, the $200 \mathrm{~m}$ world record was revised six times from $19.83 \mathrm{~s}$ to $19.19 \mathrm{~s}$ what amounts to $3.33 \%$.
Sprinting speed is defined with the frequency and the length of strides (Mann and Herman, 1985; Ae et al., 1992; Delecluse et al., 1998; Brüggemann et al., 1999; Gajer et al., 1999; Ferro et al., 2001). These parameters are mutually dependant with their optimal ratio enabling maximal sprinting speed. The increase of speed can be achieved by increased length or frequency of strides. The increase of both parameters simultaneously is quite difficult due to mutual dependency. Therefore an increase in one factor will result in an improvement in sprint velocity,

1 - University of School of Physical Education, Department of Track and Field.Wroctaw, Poland

2 - University of Jyvaskyla, Department of Biology of Physical Activity. Finland 
as long as the other factor does not undergo a proportionately similar or larger decrease (Hunter at el., 2004). Increased frequency results in shorter stride length and vice versa. Therefore the increase in stride length must be directly proportional with the decrease of stride frequency, especially at the beginning of the race the initial acceleration phase (Mackala, 2007). This relationship is individually conditioned with the processes of neuro-muscular regulation of movement, morphological characteristics, motor abilities and energy substrates (Mann and Sprague, 1980; Mero et al., 1992; Harland and Steele, 1997; Novacheck, 1998; Coh et al., 2001; Prampero et al., 2005).

According to Hunter et al. (2004) and Bezodias et al. (2008), research investigating the relative importance of developing a long stride length or a high stride rate has been inconsistent across published data. Mann and Herman (1985), Ae et al. (1992) and Bezodias et al. (2008) suggested that SF was a more important contributor to the velocity increase in sprint performance, where Mero and Komi (1985), Gajer et al. (1999), Shen (2000) and Mackala (2007) stated that SL was a more significant variable. However, it is not clear how those two kinematic parameters interact with each other across the entire distance of $100 \mathrm{~m}$ in order to accurately identify different phases of the sprint race. No data exist on how world class sprinters manipulate stride frequency and stride length in order to reach optimal efficiency of the sprint run.

The purpose of this investigation was to compare and determine the relevance of the morphological characteristics and variability of running speed parameters (stride length and stride frequency) between Usain Bolt's three best $100 \mathrm{~m}$ performances. Based on this, an attempt was made to define which factors determine the performance of Usain Bolt's sprint and, therefore, distinguish him from other sprinters.

The presented reasoning leads to the following hypotheses: the stride length is the main factor that determines the increase of running speed in particular $10 \mathrm{~m}$ sections of the entire $100 \mathrm{~m}$ distance. Knowledge of the relative influence of stride length or stride frequency on maximal running speed would be of great value to coaches and provide a basis for developing specifically designed training protocols for maximum speed development.

\section{Material and Methods}

\section{Participants}

The participants for this study were: a Jamaican sprinter, Usain Bolt (body height $=196$ $\mathrm{cm}$, body mass $=93 \mathrm{~kg}$, age $=26$ years), current $100 \mathrm{~m}$ world record holder (9.58 s) and other world class male sprinters, finalists of: 2008 Beijing Olympic Games (age $=25.3 \pm 2.93$ years, body height $=176.0 \pm 8.40 \mathrm{~cm}$, body mass $=76.7 \pm$ $6.41 \mathrm{~kg}$, and $100 \mathrm{~m}$ performance $=9.96 \pm 0.05 \mathrm{~s}$ (the best result: 9.98 s)), 2009 Berlin World Championships in Athletics (age $=26.7 \pm 4.07$ years, body height $=177.3 \pm 6.40 \mathrm{~cm}$, body mass $=$ $79.0 \pm 8.01 \mathrm{~kg}$, and $100 \mathrm{~m}$ performance $=9.91 \pm 0.10$ s (the best result: 9.71 s)), and 2012 London Olympic Games (age $=27.0 \pm 3.26$ years, body height $=179.4 \pm 8.10 \mathrm{~cm}$, body mass $=80.4 \pm 8.27$ $\mathrm{kg}$, and $100 \mathrm{~m}$ performance $=9.86 \pm 0.10 \mathrm{~s}$ (the best result: $9.75 \mathrm{~s})$ ). They were assigned to 2 groups: Usain Bolt and other finalists ( $n=7$ or $n=6)$.

\section{Anthropometric characteristics}

Body mass, body height, body mass index (BMI) and age were collected for each participant from:

a)www.2008.NBColympics.com/track/athletes for 2008 Beijing Olympic Games finalists $(n=8)$, b) www.trackandfield.about.com/profiles for 2009 Berlin World Championships in Athletics finalists $(\mathrm{n}=8)$, and c)www.BBC.uk/sport/olimpics/2012/athletes for 2012 London Olympic Games finalists $(\mathrm{n}=8)$.

The measurements take into account the changes in age and body mass, if there were such for the sprinters taking part in all three finals. Based on measurements of body mass the current BMI was established. BMI was calculated as the ratio of body mass to the squared standing stature $(\mathrm{kg} \cdot \mathrm{m}-2)$.

\section{Measures and procedures}

The data collections were conducted at the 2008 Beijing Olympic Games, 2009 Berlin World Championships in Athletics and 2012 London Olympic Games. The data was obtained from video footage provided by NBC TV stations and BBC TV station and available due to the courtesy of TV on the web. After downloading the video footage from the network in order to see the movie frame by frame, the VirtualDub 
Programme was applied. The VirtualDub Programme is a video capture/processing utility for 32-bit and 64-bit Windows platforms (98/ME/NT4/2000/XP/Vista/7), licensed under the GNU General Public License (GPL). Through this program, we developed a file format of individual frames where the frames in the video were counted. This enabled the acquisition of basic kinematic data such as: division of the $100 \mathrm{~m}$ distance into $10 \mathrm{~m}$ sections, average interval time, the number of strides performed, the average stride length calculations, average stride frequency, as well as calculation of running speed for each 10 or $20 \mathrm{~m}$ section, at the end of each 10 or $20 \mathrm{~m}$ segment. Our data for each $100 \mathrm{~m}$ final was compared with other data posted in the web or available by courtesy of the TV station. In turn, our data for the Olympic Games in Beijing in 2008 was compared with data from speedenduramce.com. However, this data analyzed only five - $20 \mathrm{~m}$ sections. The $100 \mathrm{~m}$ final in Berlin in 2009 was compared with data obtained and published by the Scientific Research Project Office responsible on behalf of the IAAF and the German Athletics Association (Berlin 2009) for a research project carried out during the competition. The data regarding $100 \mathrm{~m}$ final from 2012 London Olympic Games applies only to Usain Bolt and was published by the Spanish newspaper El Pais. The application of VirtualDub Programme allowed measurement only for a selected sprinter. Accurate measurements from a camera were possible only fore those sprinters not visually obscured or interfered by other sprinters (placement of the foot on the track). This problem occurs at the beginning of the race (acceleration phase), and between 60 and $90 \mathrm{~m}$ of the sprint. The material was read and later developed from commercial recording (TV - NBC, BBC) not from the biomechanical set-up on the stadium directly during $100 \mathrm{~m}$ performance. This may imply the inaccuracies and missing data. However, data was compared with the sources and no significant differences were found.

\section{Statistical analysis}

In the analysis descriptive statistics were applied. It included the calculation of mean, SD and V (variation). All data were analyzed using the statistics package for windows Statistical Package for Social Science (v. 11,0, Chicago Il.).

\section{Results}

Despite the lack of strong evidence (relationship) between body composition and results in the $100 \mathrm{~m}$ sprint, we decided to utilize the information of morphological characteristics of Usain Bolt and compare it with other finalists. This information could not be disregarded especially when analyzing the relationship between running speed, length and frequency of strides and the result in sprinting.

Physically, with body height of $196 \mathrm{~cm}$, Bolt is one of the tallest sprinters in the world. The current second highest sprinter is Ryan Bailey from USA of $193 \mathrm{~cm}$. For comparison, Bolt's height is almost $20 \mathrm{~cm}, 18.7 \mathrm{~cm}$ and $16.6 \mathrm{~cm}$ greater than the average height of the other finalists (2012 Beijing Olympic Games, 2009 Berlin World Championships in Athletics and 2012 London Olympic Games). On the other hand, body mass shows even greater differences between Bolt and the other sprinters. Bolt, despite greater height, is also heavier than the other sprinters: Beijing $14.8 \%$, Berlin $12.3 \%$ and $10.7 \%$ in London. The disclosed values directly reflect the level of BMI, although the differences are less pronounced (Table 1).

Table 2 contains the basic parameters of three best Usain Bolt sprint performances, compared with the rest of finalists. Bolt completed all three fastest $100 \mathrm{~m}$ races in an average of 41.13 strides, starting with smaller steps at the beginning of the sprint and covering an average of 2.45 meters with each one. His opponents took about 43-48 strides (the average was: 45.65 strides in Beijing 2008, 44.91 strides in Berlin 2009 and 44.45 strides in London 2012, which gives the average stride length for each competitions $(2.19 \mathrm{~cm}, 2.23 \mathrm{~cm}$ and $2.25 \mathrm{~cm}$ respectively)). Of course, the stride length is inextricably linked to its frequency, which is about $0.30 \mathrm{~Hz}$ lower in Usain Bolt, than in the remaining finalists. Comparing this with Bolt's times (average of three races $-9,63 \mathrm{~s}$ ) with that of his rivals (the average of three races - $9.91 \mathrm{~s}$ ) gives an opportunity to see how he is able to perfectly manage those two kinematic variables in order to reach $12,34 \mathrm{~m} / \mathrm{s}$ in some $10 \mathrm{~m}$ sections of his $100 \mathrm{~m}$ performances

Table 3 contains Usain Bolt's 100 m kinematic data with a breakdown to $10 \mathrm{~m}$ sections. This table gives us some real insight into Bolt's races. It showed that most of the times in 
each $10 \mathrm{~m}$ section from Bejing are similar to those from Berlin and London. The differences are on average of 0.02 seconds. Clear differences can be observed only in the first and last $10 \mathrm{~m}$. Despite the better reaction in comparison to the final in Beijing (a difference of $0.019 \mathrm{~s}$ ), Bolt's first $10 \mathrm{~m}$ in Berlin was slower by $0.04 \mathrm{~s}$ (total time of the first $10 \mathrm{~m}$ section was $1.89 \mathrm{~s})$ compared to his race in Beijing. The second significant difference is in the last $10 \mathrm{~m}$. It cost him about $0.07 \mathrm{~s}$ compared to the time needed for the last $10 \mathrm{~m}$ in Berlin. That is an important time difference for $10 \mathrm{~m}$. After deducting the reaction time $(0.019 \mathrm{~s})$ from the first $10 \mathrm{~m}$ section (1.85 s) performed in Beijing, we can suppose that Bolt would be able to sprint the first $10 \mathrm{~m}$ in $1.83 \mathrm{~s}$, or about $0.06 \mathrm{~s}$ faster than he did in the final event in Berlin. Thus, a new world record in the $100 \mathrm{~m}$ probably would be 9.52 seconds. In London final from 80 to 100 meters Bolt actually began to slow down. We can see that his time for the last 20 meters is 0.06 seconds slower than his fastest 20 meter split of 1.61 seconds. We also know that he reached his average maximum speed $12.34 \mathrm{~s}$ (between 60-80 m).

Table 1

Baseline physical characteristics of Usain Bolt and the rest of finalists of the $100 \mathrm{~m}$ sprint

\begin{tabular}{|c|c|c|c|c|c|c|c|c|c|}
\hline \multirow[t]{3}{*}{ Parameters } & \multicolumn{3}{|c|}{$\begin{array}{l}\text { Olympic Games } \\
\text { Beijing } 2008 \text { (1) }\end{array}$} & \multicolumn{3}{|c|}{$\begin{array}{c}\text { World Championships } \\
\text { Berlin } 2009 \text { (2) }\end{array}$} & \multicolumn{3}{|c|}{$\begin{array}{l}\text { Olympic Games } \\
\text { London } 2012 \text { (3) }\end{array}$} \\
\hline & \multirow{2}{*}{$\begin{array}{c}\text { UsainBol } \\
\mathrm{t}\end{array}$} & \multicolumn{2}{|c|}{ Other Finalists } & \multirow{2}{*}{$\begin{array}{l}\text { Usain } \\
\text { Bolt }\end{array}$} & \multicolumn{2}{|c|}{ Other Finalists } & \multirow{2}{*}{$\begin{array}{c}\text { Usain } \\
\text { Bolt }\end{array}$} & \multicolumn{2}{|c|}{ Other Finalists } \\
\hline & & $\bar{x}$ & $\mathrm{SD}$ & & $\bar{x}$ & SD & & $\bar{x}$ & $\mathrm{SD}$ \\
\hline Age (year) & 22 & 25.3 & 2.93 & 23 & 26.7 & 4.07 & 26 & 27.0 & 3.26 \\
\hline Body mass $(\mathrm{kg})$ & 90 & 76.7 & 6.41 & 90 & 79.0 & 8.01 & 93 & 80.4 & 8.27 \\
\hline Body height $(\mathrm{m})$ & 196 & 176 & 3.64 & 196 & 177.3 & 6.40 & 196 & 179.4 & 8.10 \\
\hline $\mathrm{BMI}\left(\mathrm{kg} / \mathrm{m}^{2}\right)$ & 23.4 & 25.5 & 2.34 & 23.4 & 22.5 & 2.26 & 24.2 & 24.9 & 1.58 \\
\hline $\begin{array}{l}\mathbf{1} \text { data from } w w w \\
\mathbf{2} \text { data from } w w w \\
\mathbf{3} \text { data from } w w w\end{array}$ & $\begin{array}{l}\text { S.NColymp } \\
\text { candfield.ab } \\
\text { uk/sport/oli }\end{array}$ & $\begin{array}{l}. \mathrm{com} / \mathrm{t} \\
\mathrm{com} / \mathrm{p} \\
\mathrm{ics} / 20\end{array}$ & $\begin{array}{l}\text { athlete } \\
\text { as } \\
\text { letes }\end{array}$ & & & & & & \\
\hline
\end{tabular}

Table 2

Numerical characteristics of selected kinematic parameters in the $100 \mathrm{~m}$ sprint:

a) Usain Bolt,

b) the rest of finalists

\begin{tabular}{|c|c|c|c|c|c|c|c|c|c|c|c|}
\hline \multirow{2}{*}{\multicolumn{2}{|c|}{$\begin{array}{l}\text { Kinematic parameters } \\
\text { a) Usain Bolt }\end{array}$}} & \multirow[t]{2}{*}{$\begin{array}{c}\text { Olympic } \\
\text { Games } \\
\text { Beijing } 2008\end{array}$} & \multirow{2}{*}{\multicolumn{2}{|c|}{$\begin{array}{c}\text { World } \\
\text { Championships } \\
\text { Berlin } 2009\end{array}$}} & \multirow{2}{*}{\multicolumn{2}{|c|}{$\begin{array}{c}\text { Olympic } \\
\text { Games } \\
\text { London } 2012\end{array}$}} & \multicolumn{5}{|c|}{$\begin{array}{l}\text { Statistics from three } \\
\text { competitions }\end{array}$} \\
\hline & & & & & & & $\bar{x}$ & \multicolumn{2}{|c|}{ SD } & \multicolumn{2}{|l|}{$\mathrm{V}$} \\
\hline \multicolumn{2}{|l|}{ Time [s] } & 9.69 & \multicolumn{2}{|r|}{9.58} & \multicolumn{2}{|c|}{9.63} & 9.63 & \multicolumn{2}{|c|}{0.06} & \multicolumn{2}{|l|}{0.57} \\
\hline \multicolumn{2}{|c|}{ Velocity [m/s] } & \multicolumn{2}{|c|}{10.32} & \multicolumn{2}{|l|}{10.44} & 10.38 & 10.38 & \multicolumn{2}{|c|}{0.06} & \multicolumn{2}{|l|}{0.58} \\
\hline \multicolumn{2}{|c|}{ Stride frequency $[\mathrm{Hz}]$} & 4.24 & \multicolumn{2}{|r|}{4.23} & \multicolumn{2}{|c|}{4.29} & 4.25 & \multicolumn{2}{|c|}{0.03} & \multicolumn{2}{|l|}{0.76} \\
\hline \multirow{3}{*}{$\begin{array}{l}\text { Number } \\
\text { of strides }\end{array}$} & All & 41.1 & \multicolumn{2}{|r|}{40.92} & \multicolumn{2}{|c|}{41.4} & 41.13 & \multicolumn{2}{|c|}{0.25} & \multicolumn{2}{|l|}{0.61} \\
\hline & Take -off from LL & \multicolumn{2}{|l|}{20.7} & \multicolumn{2}{|l|}{20.1} & 0.9 & 20.57 & & & 2.02 & \\
\hline & Take -off from RL & 20.4 & & 20.8 & & 0.5 & 20.57 & & & 1.01 & \\
\hline Stride leng & & 2.43 & & 2.47 & & 41 & 2.44 & & & 1.25 & \\
\hline & $\begin{array}{l}\text { parameters } \\
\text { t of finalists }\end{array}$ & & $\begin{array}{l}\text { apic Gar } \\
\text { ijing } 200 \\
\text { 7) } \mathbf{( 1 , 2 ,}\end{array}$ & & World & $\begin{array}{l}\text { ampion } \\
\text { in } 2009 \\
=7)(3)\end{array}$ & hips & & $\begin{array}{l}\text { ipic Ga } \\
\text { don } 20 \\
\text { 6) }(\mathbf{1}, \mathbf{4}\end{array}$ & $\begin{array}{l}\text { mes } \\
12 \\
5)\end{array}$ & \\
\hline & & $\bar{x}$ & SD & $\mathrm{V}$ & $\bar{x}$ & SD & $\mathrm{V}$ & $\bar{x}$ & SD & & $\mathrm{V}$ \\
\hline Time [s] & & 9.96 & 0.05 & 0.51 & 9.91 & 0.10 & 1.06 & 9.86 & 0.10 & & .93 \\
\hline Velocity $[\mathrm{m} / \mathrm{s}$ & & 10.04 & 0.05 & 0.51 & 10.09 & 0.11 & 1.01 & 10.15 & 0.09 & & .92 \\
\hline Stride freque & & 4.55 & 0.21 & 4.68 & 4.53 & 0.20 & 4.41 & 4.38 & 0.19 & & 1.44 \\
\hline Number & All & 45.69 & 2.17 & 4.75 & 44.91 & 1.77 & 3.94 & 44.45 & 1.99 & & 4.48 \\
\hline of strides & Take -off from LL & - & - & - & - & - & - & - & - & & - \\
\hline & Take -off from RL & - & - & - & - & - & - & - & - & & - \\
\hline Stride length & & 2.19 & 0.11 & 4.84 & 2.23 & 0.09 & 3.93 & 2.25 & 0.10 & & 4.50 \\
\hline
\end{tabular}


Table 3

Selected kinematic parameters (time and velocity) of Usain Bolt in the $100 \mathrm{~m}$ sprint:

a) Beijing 2008,

b) Berlin 2009 and

c) London 2012

\begin{tabular}{|c|c|c|c|c|c|c|c|c|c|c|c|c|}
\hline \multicolumn{13}{|c|}{ a) Olympic Games Beijing 2008} \\
\hline $\begin{array}{l}\text { Distance } \\
{[\mathrm{m}]}\end{array}$ & $\begin{array}{l}\text { Mean } \\
\text { time } \\
{[\mathrm{s}]^{*}}\end{array}$ & $\begin{array}{l}\text { mean } \\
\text { time } \\
{[\mathrm{s}]^{* *}}\end{array}$ & $\begin{array}{l}\text { Total } \\
\text { time }\end{array}$ & $\begin{array}{c}\text { Mean } \\
\text { velocity } \\
{[\mathrm{m} / \mathrm{s}]}\end{array}$ & $\begin{array}{c}\text { Total } \\
\text { velocity } \\
{[\mathrm{m} / \mathrm{s}]}\end{array}$ & $\begin{array}{l}\text { Number } \\
\text { of strides }\end{array}$ & $\begin{array}{l}\text { Each } \\
20 \mathrm{~m}\end{array}$ & Total & $\begin{array}{l}\text { Stride } \\
\text { length } \\
{[\mathrm{m}]}\end{array}$ & $\begin{array}{l}\text { Each } \\
20 \mathrm{~m}\end{array}$ & $\begin{array}{c}\text { Stride } \\
\text { frequency } \\
{[\mathrm{Hz}]}\end{array}$ & $\begin{array}{l}\text { Each } \\
20 \mathrm{~m}\end{array}$ \\
\hline $0-10$ & 1.85 & 1.89 & 1.85 & 5.40 & 5.40 & 7.0 & \multirow[t]{2}{*}{11.1} & \multirow[t]{2}{*}{11.0} & 1.41 & \multirow[t]{2}{*}{1.82} & 3.84 & \multirow[t]{2}{*}{3.83} \\
\hline $10-20$ & 1.02 & 0.99 & 2.87 & 9.80 & 6.97 & 4.1 & & & 2.44 & & 4.01 & \\
\hline $20-30$ & 0.91 & 0.90 & 3.78 & 10.99 & 7.94 & 4.0 & \multirow[t]{2}{*}{8.0} & \multirow[t]{2}{*}{19.0} & 2.50 & \multirow[t]{2}{*}{2.50} & 4.39 & \multirow[t]{2}{*}{4.49} \\
\hline $30-40$ & 0.87 & 0.86 & 4.65 & 11.49 & 8.60 & 3.9 & & & 2.56 & & 4.48 & \\
\hline $40-50$ & 0.85 & 0.83 & 5.50 & 11.76 & 9.09 & 3.9 & \multirow[t]{2}{*}{7.6} & \multirow[t]{2}{*}{26.6} & 2.56 & \multirow[t]{2}{*}{2.63} & 4.59 & \multirow[t]{2}{*}{4.55} \\
\hline $50-60$ & 0.82 & 0.82 & 6.32 & 12.19 & 9.49 & 3.7 & & & 2.70 & & 4.51 & \\
\hline $60-70$ & 0.82 & 0.81 & 7.14 & 12.19 & 9.80 & 3.8 & \multirow[t]{2}{*}{7.4} & \multirow[t]{2}{*}{34.0} & 2.63 & \multirow[t]{2}{*}{2.70} & 4.63 & \multirow[t]{2}{*}{4.51} \\
\hline $\begin{array}{l}70-80 \\
\end{array}$ & 0.82 & 0.82 & 7.96 & 12.19 & 10.05 & 3.6 & & & 2.77 & & 4.39 & \\
\hline $80-90$ & 0.83 & 0.83 & 8.79 & 12.05 & 10.24 & 3.5 & \multirow[t]{2}{*}{7.1} & \multirow[t]{2}{*}{41.1} & 2.86 & \multirow[t]{2}{*}{2.83} & 4.22 & \multirow[t]{2}{*}{4.10} \\
\hline $90-100$ & 0.90 & 0.83 & 9.69 & 11.11 & 10.31 & 3.6 & & & 2.77 & & 4.00 & \\
\hline
\end{tabular}

\begin{tabular}{|c|c|c|c|c|c|c|c|c|c|c|c|c|}
\hline \multicolumn{13}{|c|}{ b) World Championship - Berlin 2009} \\
\hline $\begin{array}{c}\text { Distance } \\
{[\mathrm{m}]}\end{array}$ & $\begin{array}{l}\text { Mean } \\
\text { time } \\
{[\mathrm{s}]^{*}}\end{array}$ & $\begin{array}{c}\text { Mean } \\
\text { time } \\
{[\mathrm{s}]^{* *}}\end{array}$ & $\begin{array}{l}\text { Total } \\
\text { time }\end{array}$ & $\begin{array}{l}\text { Mean } \\
\text { velocity } \\
{[\mathrm{m} / \mathrm{s}]}\end{array}$ & $\begin{array}{c}\text { Total } \\
\text { velocity } \\
{[\mathrm{m} / \mathrm{s}]}\end{array}$ & $\begin{array}{l}\text { Number } \\
\text { of strides }\end{array}$ & $\begin{array}{c}20 \mathrm{~m} \\
\text { Section }\end{array}$ & Total & $\begin{array}{c}\text { Stride } \\
\text { length } \\
{[\mathrm{m}]}\end{array}$ & $\begin{array}{l}\text { Each } \\
20 \mathrm{~m}\end{array}$ & $\begin{array}{c}\text { Stride } \\
\text { frequency } \\
{[\mathrm{Hz}]}\end{array}$ & $\begin{array}{l}\text { Each } \\
20 \mathrm{~m}\end{array}$ \\
\hline $0-10$ & 1.89 & 1.90 & 1.90 & 5.26 & 5.26 & 7 & \multirow[t]{2}{*}{11.2} & \multirow[t]{2}{*}{11.2} & 1.43 & \multirow[t]{2}{*}{1.78} & 3.68 & \multirow[t]{2}{*}{3.89} \\
\hline $10-20$ & 0.99 & 0.98 & 2.88 & 10.20 & 6.94 & 4.2 & & & 2.38 & & 4.28 & \\
\hline $20-30$ & 0.90 & 0.92 & 3.80 & 10.87 & 7.90 & 4.0 & \multirow[t]{2}{*}{7.9} & \multirow[t]{2}{*}{19.1} & 2.50 & \multirow[t]{2}{*}{2.52} & 4.35 & \multirow[t]{2}{*}{4.54} \\
\hline $30-40$ & 0.86 & 0.83 & 4.63 & 12.05 & 8.64 & 3.9 & & & 2.56 & & 4.70 & \\
\hline $40-50$ & 0.83 & 0.84 & 5.46 & 11.90 & 9.16 & 3.8 & \multirow[t]{2}{*}{7.5} & \multirow[t]{2}{*}{26.6} & 2.63 & \multirow[t]{2}{*}{2.67} & 4.64 & \multirow[t]{2}{*}{4.49} \\
\hline $50-60$ & 0.82 & 0.82 & 6.29 & 12.19 & 9.53 & 3.8 & & & 2.63 & & 4.63 & \\
\hline $60-70$ & 0.81 & 0.82 & 7.11 & 12.19 & 9.84 & 3.7 & \multirow[t]{2}{*}{7.2} & \multirow[t]{2}{*}{33.8} & 2.70 & \multirow[t]{2}{*}{2.77} & 4.51 & \multirow[t]{2}{*}{4.49} \\
\hline 70-80 & 0.82 & 0.81 & 7.92 & 12.34 & 10.10 & 3.5 & & & 2.86 & & 4.32 & \\
\hline $80-90$ & 0.83 & 0.83 & 8.75 & 12.05 & 10.28 & 3.4 & \multirow[t]{2}{*}{7.0} & \multirow[t]{2}{*}{40.8} & 2.94 & \multirow[t]{2}{*}{2.85} & 4.10 & \multirow[t]{2}{*}{4.23} \\
\hline $90-100$ & 0.83 & 0.83 & 9.58 & 12.05 & 10.44 & 3.6 & & & 2.77 & & 4.34 & \\
\hline
\end{tabular}

\begin{tabular}{|c|c|c|c|c|c|c|c|c|c|c|c|c|}
\hline \multicolumn{13}{|c|}{ c) Olympic Games - London 2012} \\
\hline $\begin{array}{c}\text { Distance } \\
{[\mathrm{m}]}\end{array}$ & $\begin{array}{c}\text { Average } \\
\text { time } \\
{[\mathrm{s}]^{*}}\end{array}$ & $\begin{array}{c}\text { Total } \\
\text { time } \\
{[\mathrm{s}]}\end{array}$ & $\begin{array}{l}\text { Total } \\
\text { time } \\
{[\mathrm{s}]^{* *}}\end{array}$ & $\begin{array}{c}\text { Average } \\
\text { velocity } \\
{[\mathrm{m} / \mathrm{s}]}\end{array}$ & $\begin{array}{c}\text { Total } \\
\text { velocity } \\
{[\mathrm{m} / \mathrm{s}]}\end{array}$ & $\begin{array}{c}\text { Number } \\
\text { of strides }\end{array}$ & $\begin{array}{c}20 \mathrm{~m} \\
\text { Section }\end{array}$ & $\begin{array}{c}\text { Tota } \\
1\end{array}$ & $\begin{array}{c}\text { Stride } \\
\text { length } \\
{[\mathrm{m}]}\end{array}$ & $\begin{array}{l}\text { Each } \\
20 \mathrm{~m}\end{array}$ & $\begin{array}{c}\text { Stride } \\
\text { frequency } \\
{[\mathrm{Hz}]}\end{array}$ & $\begin{array}{l}\text { Each } \\
20 \mathrm{~m}\end{array}$ \\
\hline $0-10$ & 1.91 & 1.91 & & 5.23 & 5.23 & 7.3 & \multirow[t]{2}{*}{11.5} & \multirow[t]{2}{*}{11.5} & 1.37 & \multirow[t]{2}{*}{1.74} & 3.82 & \multirow[t]{2}{*}{3.94} \\
\hline $10-20$ & 1.01 & 2.92 & 2.93 & 9.90 & 6.84 & 4.2 & & & 2.38 & & 4.16 & \\
\hline $20-30$ & 0.92 & 3.84 & & 10.87 & 7.81 & 4.1 & \multirow[t]{2}{*}{8.0} & \multirow[t]{2}{*}{19.5} & 2.44 & \multirow[t]{2}{*}{2.50} & 4.45 & \multirow[t]{2}{*}{4.49} \\
\hline $30-40$ & 0.86 & 4.70 & 4.69 & 11.63 & 8.51 & 3.9 & & & 2.56 & & 4.54 & \\
\hline $40-50$ & 0.84 & 5.54 & & 11.90 & 9.02 & 4.0 & \multirow[t]{2}{*}{7.8} & \multirow[t]{2}{*}{27.3} & 2.50 & \multirow[t]{2}{*}{2.56} & 4.76 & \multirow[t]{2}{*}{4.70} \\
\hline $50-60$ & 0.82 & 6.36 & 6.35 & 12.19 & 9.43 & 3.8 & & & 2.63 & & 4.63 & \\
\hline $60-70$ & 0.81 & 7.17 & & 12.34 & 9.76 & 3.6 & \multirow[t]{2}{*}{7.0} & \multirow[t]{2}{*}{34.3} & 2.78 & \multirow[t]{2}{*}{2.86} & 4.44 & \multirow[t]{2}{*}{4.32} \\
\hline $70-80$ & 0.81 & 7.98 & 7.96 & 12.34 & 10.02 & 3.4 & & & 2.94 & & 4.20 & \\
\hline $80-90$ & 0.82 & 8.80 & & 12.19 & 10.23 & 3.6 & \multirow[t]{2}{*}{7.1} & \multirow[t]{2}{*}{41.4} & 2.78 & \multirow[t]{2}{*}{2.82} & 4.38 & \multirow[t]{2}{*}{4.30} \\
\hline $90-100$ & 0.83 & 9.63 & 9.63 & 12.05 & 10.38 & 3.5 & & & 2.86 & & 4.21 & \\
\hline
\end{tabular}

* Data from VirtualDub

** Data from El Pais 
Table 4

Comparison of the $20 \mathrm{~m}$ sections time interval, speed, stride length, stride frequency and stride numbers between Usain Bolt

and the rest of athletes of the $100 \mathrm{~m}$

final of World Championship - Berlin 2009

\begin{tabular}{|c|c|c|c|c|c|c|c|c|c|c|c|c|c|c|c|}
\hline \multicolumn{6}{|c|}{ Usain Bolt - World Championship - Berlin 2009} & \multicolumn{10}{|c|}{ The Rest of Finalists - World Championship - Berlin 2009} \\
\hline \multirow[t]{2}{*}{$\begin{array}{l}\text { Distance } \\
\text { (m) }\end{array}$} & \multirow[t]{2}{*}{$\begin{array}{l}\text { Total } \\
\text { Time } \\
\text { (s) }\end{array}$} & \multirow[t]{2}{*}{$\begin{array}{c}\text { Mean } \\
\text { velocity } \\
{[\mathrm{m} / \mathrm{s}]}\end{array}$} & \multirow[t]{2}{*}{$\begin{array}{c}\text { Mean } \\
\text { stride } \\
\text { numbers }\end{array}$} & \multirow{2}{*}{$\begin{array}{c}\text { Mean } \\
\text { stride } \\
\text { length } \\
(\mathrm{m})\end{array}$} & \multirow{2}{*}{$\begin{array}{c}\text { Mean } \\
\text { stride } \\
\text { frequency } \\
(\mathrm{Hz})\end{array}$} & \multicolumn{2}{|c|}{$\begin{array}{l}\text { Total } \\
\text { time (s) }\end{array}$} & \multicolumn{2}{|c|}{$\begin{array}{l}\text { Mean } \\
\text { velocity } \\
{[\mathrm{m} / \mathrm{s}]}\end{array}$} & \multicolumn{2}{|c|}{$\begin{array}{c}\text { Mean } \\
\text { stride } \\
\text { numbers }\end{array}$} & \multicolumn{2}{|c|}{$\begin{array}{l}\text { Mean stride } \\
\text { length } \\
\text { (m) }\end{array}$} & \multicolumn{2}{|c|}{$\begin{array}{l}\text { Mean stride } \\
\text { frequency } \\
(\mathrm{Hz})\end{array}$} \\
\hline & & & & & & $\overline{\bar{x}}$ & SD & $\overline{\bar{x}}$ & SD & $\overline{\bar{x}}$ & SD & $\overline{\bar{x}}$ & SD & $\overline{\bar{x}}$ & SD \\
\hline $0-20$ & 2.88 & 6.94 & 11.2 & 1.78 & 3.89 & 2.93 & 0.02 & 6.83 & $\begin{array}{c}0.0 \\
5\end{array}$ & 12.09 & 0.51 & 1.66 & 0.07 & 4.13 & 0.18 \\
\hline $20-40$ & 1.75 & 11.42 & 7.9 & 2.52 & 4.54 & 1.81 & 0.01 & 11.06 & $\begin{array}{c}0.0 \\
9\end{array}$ & 8.73 & 0.37 & 2.29 & 0.10 & 4.84 & 0.23 \\
\hline $40-60$ & 1.66 & 12.05 & 7.5 & 2.67 & 4.49 & 1.73 & 0.02 & 11.54 & $\begin{array}{c}0.1 \\
7\end{array}$ & 8.21 & 0.31 & 2.44 & 0.09 & 4.75 & 0.20 \\
\hline $60-80$ & 1.63 & 12.26 & 7.2 & 2.77 & 4.49 & 1.70 & 0.03 & 11.80 & $\begin{array}{c}0.2 \\
3\end{array}$ & 8.06 & 0.27 & 2.48 & 0.08 & 4.77 & 0.20 \\
\hline $80-100$ & 1.66 & 12.05 & 7.0 & 2.85 & 4.23 & 1.75 & 0.03 & 11.43 & $\begin{array}{c}0.2 \\
0\end{array}$ & 7.81 & 0.45 & 2.56 & 0.14 & 4.49 & 0.30 \\
\hline
\end{tabular}

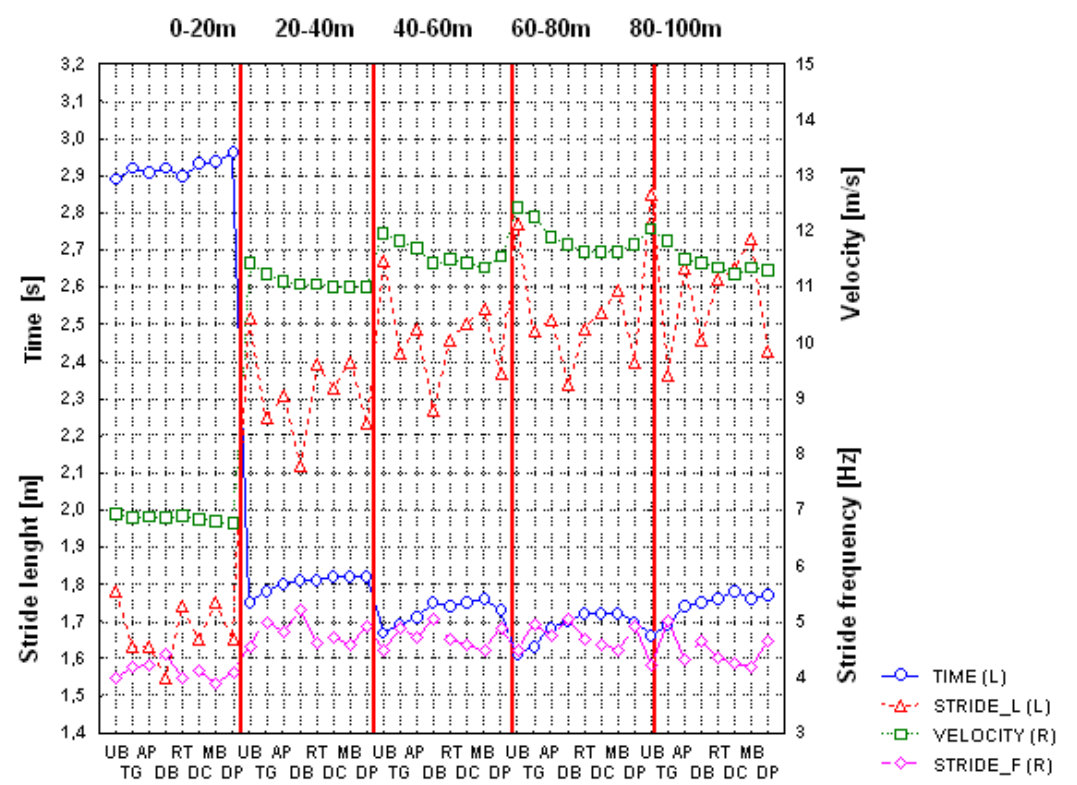

Figure 1

Individual characteristics of groups of selected kinematic parameters from the $100 \mathrm{~m}$ final of 2009 Berlin World Championships in Athletics 
Table 5

Differences in interval speed, stride length, stride frequency of Usain Bolt (World Record) in:

a) Olympic Games in Beijing 2008,

b) World Championships in Berlin 2009 and

c) Olympic Games in London 2012

\begin{tabular}{|c|c|c|c|c|c|c|c|c|c|c|}
\hline \multirow[t]{2}{*}{ Descriptive statistic } & \multicolumn{10}{|c|}{ a) Olympic Games Beijing 2008} \\
\hline & 10 & 20 & 30 & 40 & 50 & 60 & 70 & 80 & 90 & 100 \\
\hline Absolute change in speed $(\mathrm{m} / \mathrm{s})$ & & 4.40 & 1.19 & 0.50 & 0.27 & 0.43 & 0.00 & 0.00 & 0.14 & 0.94 \\
\hline \multirow[t]{2}{*}{ Absolute change in stride length $(\mathrm{m})$} & 1.41 & $(+)$ & $(+)$ & $(+)$ & $(+)$ & $(+)$ & $(-)$ & $(+)$ & $(+)$ & $(-)$ \\
\hline & & 1.03 & 0.06 & 0.06 & 0.00 & 0.14 & 0.07 & 0.14 & 0.09 & 0.09 \\
\hline \multirow[t]{2}{*}{ Absolute change in stride frequency $(\mathrm{Hz})$} & 3.84 & $(+)$ & $(+)$ & $(+)$ & $(+)$ & $(-)$ & $(+)$ & $(-)$ & $(-)$ & $(-)$ \\
\hline & & 0.17 & 0.38 & 0.09 & 0.11 & 0.08 & 0.12 & 0.24 & 0.17 & 0.22 \\
\hline Relative change in stride frequency (\%) & 0.0 & 4.4 & 9.5 & 2.0 & 2.4 & 1.7 & 2.7 & 5.2 & 3.9 & 5.2 \\
\hline
\end{tabular}

$(+)$ increase, $(-)$ decrease, ${ }^{*}$ Data from VirtualDub

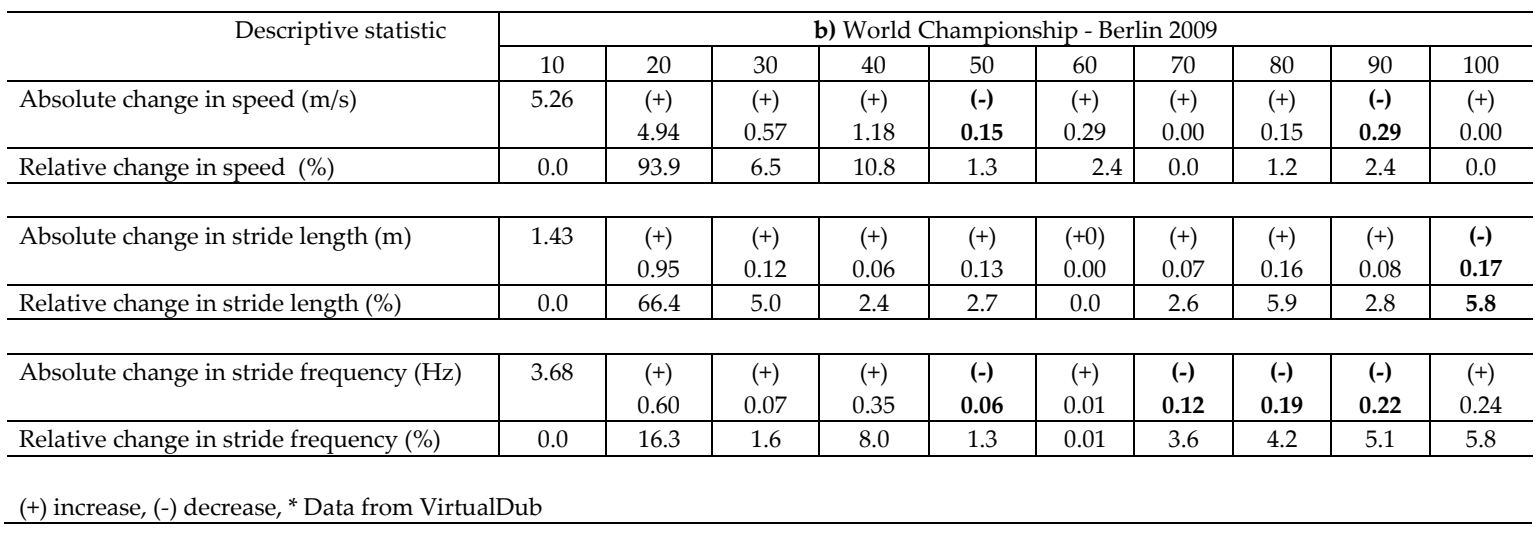

\begin{tabular}{|c|c|c|c|c|c|c|c|c|c|c|}
\hline \multirow[t]{2}{*}{ Descriptive statistic } & \multicolumn{10}{|c|}{ c) Olympic Games - London 2012} \\
\hline & 10 & 20 & 30 & 40 & 50 & 60 & 70 & 80 & 90 & 100 \\
\hline Absolute change in speed $(\mathrm{m} / \mathrm{s})$ & 5.23 & $(+)$ & $(+)$ & $(+)$ & $(+)$ & $(+)$ & $(+)$ & $(=)$ & $(-)$ & $(-)$ \\
\hline & & 4.67 & 0.97 & 0.76 & 0.27 & 0.29 & 0.15 & 0.00 & 0.15 & 0.14 \\
\hline Relative change in speed (\%) & 0.0 & 89.3 & 9.8 & 6.9 & 2.3 & 2.4 & 1.2 & 0.0 & 1.2 & 1.2 \\
\hline
\end{tabular}

\begin{tabular}{|c|c|c|c|c|c|c|c|c|c|c|}
\hline Relative change in stride length (\%) & 0.0 & 73.7 & 5.7 & 4.9 & 2.4 & 5.2 & 5.7 & 5.7 & 5.5 & 2.9 \\
\hline Absolute change in stride frequency $(\mathrm{Hz})$ & 3.82 & $\begin{array}{c}(+) \\
0.34\end{array}$ & $\begin{array}{c}(+) \\
0.29\end{array}$ & $\begin{array}{c}(+) \\
0.09\end{array}$ & $\begin{array}{c}+(+) \\
0.22\end{array}$ & $\begin{array}{c}(-) \\
0.13\end{array}$ & $\begin{array}{c}(-) \\
0.19\end{array}$ & $\begin{array}{c}(-) \\
0.24\end{array}$ & $\begin{array}{c}(+) \\
0.18\end{array}$ & $\begin{array}{c}(-) \\
0.17\end{array}$ \\
\hline Relative change in stride frequency (\%) & 0.0 & 8.9 & 7.0 & 2.0 & 4.8 & 2.7 & 4.1 & 5.4 & 4.3 & 4.9 \\
\hline
\end{tabular}

$\underline{(+) \text { increase, }(-) \text { decrease, }{ }^{*} \text { Data from VirtualDub }}$ 
To better illustrate the differences between Usain Bolt and other world-class sprinters, Table 4 and Figure 1 compared the kinematic fundamental values of the $100 \mathrm{~m}$ final in 2009 from Berlin divided into $20 \mathrm{~m}$ sections. The individual characteristics of selected kinematic parameters in 100 sprint showed significant differences between sprinters.

Table 5 contains the basic criterion for determining the effectiveness of the speed curve of $100 \mathrm{~m}$ sprint. The changes of speed value depend on mutual relations between the stride length and stride frequency. It is evident that up to $40 \mathrm{~m}$ at the same time the running speed increases, due to a linear increase in both the stride length and stride frequency. In the later part of the distance, there is a further increase in velocity, although the length and frequency demonstrated a large variable.

\section{Discussion}

The purpose of this investigation was to compare and determine the relevance of the morphological characteristics and variability of running speed parameters (stride length and stride frequency) between Usain Bolt's three best $100 \mathrm{~m}$ performances. Based on this, an attempt was made to define which factors determine the performance of Usain Bolt's sprint and, therefore, distinguish him from other sprinters.

The influence of Usain Bolt's biological attributes (body height and body mass) on the stride length and stride frequency would be a simplified explanation for his superiority and elements that significantly distinguish him from rest of the finalists. With regard to the body build, type of muscles (dominated by fast-twitch muscle fibers) and training program, there must be some variables which distinguish Bolt from the rest of current world sprinters. Taking into consideration that in sprinting, there are numerous limiting factors such as gravity, ground contact time, muscle build, power that generates the speed of muscle contractions, it may be assumed that some of these variables make him a super athlete, much faster than other sprinters .

Therefore, it is worth noting that Bolt's body height and long lower extremities may be also perceived as a disadvantage taking into consideration the fact that he is much slower than his shorter opponents at the acceleration phase.
On the other hand, longer lower limbs enable Bolt to propel him in the phase with maximum speed $(50-80 \mathrm{~m})$ and allow him to maintain his speed till the end of the race. Bolt's body height resulting in long strides makes it possible for him to maintain high speed for a longer time and decelerate at a slower rate than shorter sprinters.

On the contrary, fast sprinters are able to achieve higher running speed by striking the ground with greater force and much quicker than slower sprinters do. This is probably another mechanical element that distinguishes Usain Bolt from other world class sprinters. How hard and how quickly do elite sprinters strike the ground? Back to Weyand et al. (2000), at top speed, every sprinter takes around a third of a second to pick their foot up and put it down again. As we know sprinting speed is largely determined by how much force a sprinter can apply to the ground. Generally, they have two possibilities to sprint faster: strike the ground during the contact phase harder or exert the same force over a longer period of time. Considering these two options, we can partly explain why Bolt is superior compared to other sprinters. He is probably able to strike the ground more forcefully than the rest of sprinters, relative to their body mass, therefore, he might maximize his time on the ground. If we consider the option to exert the same force over a longer period of time, it can generate more power so it increases stride length. His forefoot lands probably a little bit further in front of the knee than in other sprinters, although, it does place tremendous stress on the hamstrings muscles. Bolt has an efficient force generation through stride thrust, greater hip flexibility and is able to sprint faster than other sprinters who had faster stride rates. He is biomechanically superior in leg/hip movement then other sprinters. Despite his body height, he is able to manage body strength during the start and initial acceleration phase to create greater force against the ground. It is necessary to understand that visually this type of sprinting technique is common for most top class sprinters. The differences are very small, almost invisible, what creates the impression that Usain Bolt is not only faster, but also he applies a more efficient technique. However, the lack of details with regard to kinetic data concerning these assumptions does not preclude scientifically reasonable considerations that Usain Bolt 
distinguishes himself from other world class sprinters.

Running velocity is the product of stride length and stride frequency $(v=l x f)$ (Luhtanen and Komi, 1978; Mero and Komi, 1985). According to Ballereich (1976), an increase in average velocity (v) and, therefore, a decrease in running time $(t)$ for a given distance, can only result from changes of these two parameters: an increase of stride length (with a decrease of number of strides and their frequency) or inversely, a decrease of stride length (with an increase in stride frequency) (Mackala, 2007). In turn, Delecluse et al. (1998) found a linear relationship between the length of the stride and speed. However their research did not find a significant correlation between stride frequency and speed. On the basis of the Ballereich's (1976) assumption, improving performance in the sprint events depends on five logical possibilities:

1. $V+V=(L+L) \quad f$ where $(f \sim$ constant $)$

2. $V+\quad V=L \quad(f+f)$ where $(\mathrm{L} \sim$ constant $)$

3. $V+V=(L+L) x(f+f)$

4. $V+V=(L+L) x(f-f) ;[(L+L) x(f-f)>$ L f $]$

5. $V+V=(L-L) x(f+f) ;[(L-L) x(f+f)>$ L f $]$

Analyzing Table 6, it can be concluded that running speed increased in 21 of 30 analyzed $10 \mathrm{~m}$ sections, which represents $70 \%$ of their total number. The decline rate was observed in 5 sections (16\%), and remaining 4 sections showed constant velocity $(13.3 \%)$. In half segments $(46.7 \%$ of all cases), running speed increased (10, 20, 30, $40,60,70 \mathrm{~m})$ due to the increase of both kinematic parameters of the sprint $\mathrm{V}+\mathrm{V}=(\mathrm{L}+\mathrm{L}) \mathrm{x}(\mathrm{f}+\mathrm{f})$. With these 7 cases, the length of the stride dominated over the increase of stride frequency, while in 1 case it was the opposite. In 4 cases where the speed was constant compared to previous section, stride length increased three times and stride frequency only once. The decline rate was observed in 5 sections, except for the fact that in three sections, an increase in the length of steps may be noted $\mathrm{V}-\mathrm{V}=(\mathrm{L}+\mathrm{L}) \mathrm{x}(\mathrm{f}-\mathrm{f})$ and in remaining two sections, an increase occurred in stride frequency $\mathrm{V}-\mathrm{V}=(\mathrm{L}-\mathrm{L}) \mathrm{x}(\mathrm{f}+\mathrm{f})$.

Similar results were presented in early Mackala's study (2007), which compared the finalists from WC in Tokyo (1991) with the average sprinters. In the final conclusion, the length of stride was a decisive factor with regard to the increase of running speed in each $10 \mathrm{~m}$ section. Gajer et al. (1999), who analyzed French best sprinters found that SL was a more important factor contributing to the increase in velocity in sprint performance. Opposite to this statement, Bezodis et al. (2008) conducted a longitudinal case study of stride characteristics in a world class sprinter where the changes in speed occurred as a consequence of changes in stride frequency. This confirms findings of Hunter (2004), who created a map of positive and negative interactions between stride length and stride frequency. He referred to the negative effect that an increase in step length might have on the step rate, and vice versa, as a "negative interaction". Some authors suggest that stride frequency (Mero et al., 1981; Mero et al., 1992), and related aspects (van Schenau et al., 1994; Wood, 1987) are the speed limiting factors in sprint running; whereas others (Amstrong et al., 1981; Summers, 1997; Shen, 2000; Hunter et al., 2004) indicate that a long stride is more important.

\begin{tabular}{|c|c|c|c|c|}
\hline \multicolumn{5}{|c|}{$\begin{array}{l}\text { Table } 6 . \\
\text { Comparison of the velocity curve for selected phases } \\
\text { sprint due to individual changes of the stride rate and stride length }\end{array}$} \\
\hline Phase & $\begin{array}{c}\text { Distance } \\
(\mathrm{m})\end{array}$ & $\begin{array}{c}\text { Olympic Games } \\
\text { Beijing } 2008 \\
\end{array}$ & $\begin{array}{l}\text { World Championship } \\
\text { Berlin } 2009\end{array}$ & $\begin{array}{c}\text { Olympic Games } \\
\text { Berlin } 2012\end{array}$ \\
\hline 1 & $0-10$ & $V+V=(L+L) x(f+f)$ & $V+\quad V=(L+L) x(f+f)$ & $V+\quad V=(L+L) x(f+f)$ \\
\hline 2 & $10-20$ & $V+V=(L+L) x(f+f)$ & $V+\quad V=(L+L) x(f+f)$ & $V+\quad V=(L+L) x(f+f)$ \\
\hline 3 & $20-30$ & $V+V=(L+L) x(f+f)$ & $V+\quad V=(L+L) x(f+f)$ & $V+\quad V=(L+L) x(f+f)$ \\
\hline 4 & $30-40$ & $V+V=(L+L) x(f+f)$ & $V+\quad V=(L+L) \quad x(f+f)$ & $V+\quad V=(L+L) x(f+f)$ \\
\hline 5 & $40-50$ & $V+V=(L+L) x(f+f)$ & $V-\quad V=(L+L) x(f-f)$ & $V+V=(L-L) x(f-+f)$ \\
\hline 6 & $50-60$ & $V+V=(L+L) x(f-f)$ & $V+\quad V=(L+L) x(f+f)$ & $V+\quad V=(L+L) x(f-f)$ \\
\hline 7 & $60-70$ & $\mathrm{~V}=$ const $=(L-L) x(f+f)$ & $V+V=(L+L) x(f-f)$ & $V+V=(L+L) x(f-f)$ \\
\hline 8 & $70-80$ & $\mathrm{~V}=\mathrm{const}=(L+L) x(f-f)$ & $V+V=(L+L) x(f-f)$ & $\mathrm{V}=\mathrm{const}=(L+L) x(f-\quad f)$ \\
\hline 9 & $80-90$ & $V-\quad V=(L+L) x(f-f)$ & $V+\quad V=(L+L) x(f-f)$ & $V-V=(L-L) x(f+f)$ \\
\hline 10 & $90-100$ & $V-\quad V=(L-L) x(f-f)$ & $\mathrm{V}=$ const $(L-L) x(f+f)$ & $V-V=(L+L) x(f-f)$ \\
\hline
\end{tabular}


In the present study, the finding confirming the prevalence of stride length over stride frequency was in line with other studies, nevertheless, it should be highlighted that there is a necessity to develop both, a greater stride rate and stride length (Kunz and Kaufman, 1981; Man et al., 1984; Delecluse et al., 1998). The explanation of this phenomenon was discussed by Hunter et al. (2004) who stated that a longer SL is achieved through long term development of strength and power, while SF may be the key factor in developing greater velocity.

According to the authors of this paper, the possibility of a negative or positive interaction between the two discussed kinematic parameters i.e., stride length and stride rate, is rather limited, even though such a statement may be in contradiction with other studies' results. Furthermore, when considering this issue, it is important to know how an improvement of one factor (i.e., stride length or stride rate) may affect another (Hunter et al., 2000). The main aim consists of reaching an optimal value of both parameters in order to maintain maximal running speed through the entire distance of $100 \mathrm{~m}$. Some results from Table 6 indicate that the sprinter reached optimum relationship (interaction) between stride length and stride frequency. Both variables showed no change in the values;
Vconst $=(L+\Delta L) \cdot(f-\Delta f)$. This observation should be considered when training an athlete in sprinting.

\section{Conclusions}

Bolt's anthropometric advantage (body height and lower limbs length) is not questionable and it is one of the factors that makes him faster than the rest of the finalists of each of the three discussed sprinting events. Additionally, Bolt's almost $20 \mathrm{~cm}$ longer stride presents an important benefit in the latter part of the race. Despite these factors, he is probably able to strike the ground more forcefully than other sprinters, relatively to their body mass and, therefore, he might maximize the time of the contact with the ground and apply the same force over this period of time. This ability, combined with longer stride, allows him to reach very high running speed - over 12 $\mathrm{m} / \mathrm{s}(12.05-12.34 \mathrm{~m} / \mathrm{s})$ in some $10 \mathrm{~m}$ sections of his three $100 \mathrm{~m}$ performances.

Analysis of the obtained results of this particular sprinter may be of great importance for trainers and coaches as it implies work on stride frequency (SF) in order to reach a higher value of maximal sprinting speed. Therefore, it is noteworthy that the main focus should be on the optimal interaction between stride length and stride frequency.

\section{References}

Ae M, Ito A, Suzuki M. The men's 100 meters. Scientific Research Project at the III World Championship in Athletics, Tokyo 1991. New Studies in Athletics, 1992; 7: 47-52

Armstrong L, Costil LD, Gehlse D. Biomechanical comparison of university sprinters and marathon runners. Track Tec, 1984; 87:2781-2782

Athletes anthropometric data. 2008 Available at: www. 2008.NBColympics.com/track/athletes; accessed on 22.11.2012

Athletes anthropometric data. 2012. Available at: www.trackandfield.about.com/profiles; accessed on 22.11.2012

Athletes anthropometric data. 2012. Available at: www.BBC.uk/sport/olimpics/2012/athletes; accessed on 23.11.2012

Ballreich R. Model for estimating the influence of stride length and stride frequency on time in sprinting events. [w:] Komi PV(red.) Biomechanics V-B. University Park Press, Baltimore, 1976; 208-212

Bezodis IM, Salo AI, Kerwin DG. A longitudinal case study of step characteristics in a world class sprint athlete. ISBS Conference 2008, Seoul, Korea, 2008; 537-540

Brüggemann GP, Koszewski D, Müller H. Biomechanical Research Project. Athens 1997, Final report. Meyer \& Meyer Sport, Oxford, 1999; 12-41 
Coh M., Milanovic D, Kampmiller T. Morphologic and kinematic characteristics of elite sprinters. Coll. Antropol, 2001, 25; 2: 605-610

Delecluse Ch, Ponnet H, Diels R. Stride characteristics related to running velocity in maximal sprint running. [w:] Riehle HJ, Vieten MM. (red) Proceedings II of XVI International Symposium on Biomechanics in Sports, ISBS, 1998; 146-148

Doscher W. The Art of Sprinting: Techniques for Speed and Performance. McFarland \& Company Inc. Publisher. North Carolina. USA; 151-153. 2009

Faccioni A. Kinematics data. 2009. Available at: www. adrian.sport.com; accessed on 22.11.2012

Ferro A, Rivera A, Pagola I. Biomechanical analysis of the $7^{\text {th }}$ IAAF World Championships in Athletics Seville 1999.New Studies in Athletics, 2001; (1,2): 25-60

Gajer B, Thepaut-Mathieu C, Lehenaff D. Evolution of stride and amplitude during course of the $100 \mathrm{~m}$ event in athletics. New Studies in Athletics, 1999; 3, 43-50

Harland M, Steele J. Biomechanics of the Sprint Start. Sports Med, 1997; 23 (1): 11-20

Hunter JP, Marshall RN, McNair PJ. Interaction of step length and step rate during sprint running. Med Sci Sport Exer, 2004; 36: 261-271

Kunz H, Kauffman DA. Biomechanical analysis of sprinting: decathletes versus champions. Br J Sports Med, 1981; 15:177-181

Lee J. 2008. Usain Bolt 100 meter splits and speed endurance. Available at: http://www.speedendurance.com; accessed on 11.08 .2008

Luhtanen R, Komi PV. Mechanical factors influencing running speed. [w:] Assmussen, Jorgensen (red.), Biomechanics VI-B. International series on Biomechanics, 2 B, 1978; 23-29

Mackala K. Optimisation of performance through kinematic analysis of the different phases of the 100 meters. New Studies in Athletics, 2007; 22(2): 7-16

Mann R, Sprague P. A kinetic analysis of the ground leg during sprint running. Res Q Exercise Sport, 1980; 51: 334-348

Mann R, Herman J. Kinematics analysis of Olympic sprint performance: men's 200 meters. Int J Sport Biomech 1985, 1: 151-162

Mero A, Komi PV. Effect of supramaximal velocity on biomechanical variables in sprinting. Int J Sport Biomech 1985; 1: 240-252

Mero A, Komi PV, Gregor RJ. Biomechanics of sprint running. Sports Med, 1992; 13: 376-392

Mero A, Luhtanen P, Susanka P. Kinematics of top sprint $(400 \mathrm{~m})$ running in fatigue conditions, Track and Field Quarterly Review, 1988; 1: 42-45

Mero A, Luhtanen P, Viitasalo JT, Komi PV. Relationships between the maximal running velocity, muscle fiber characteristics, force production and force relaxation of sprinters. Scand J Sports Sci, 1981; 3:16-22

Novacheck T. The biomechanics of running, Gait Posture, 1998; 7: 77-95

Prampero P, Fusi S, Sepulcri J, Morin B, Belli A, Antonutto G. Sprint running: a new energetic approach. J Exp Biol, 2005; 208: 2809-2816

Scientific Research Project 2011. IAAF, (DVL) - World Championship in Athletics, Berlin, Germany. Available at: www. iaaf.org; accessed on 10.02.2012

Shen $\mathrm{W}$. The effects of stride length and frequency on the speeds of elite sprinters in 100 meter dash, 
Biomechanical Proceedings of XVIII International Symposium of Biomechanics in Sports, HongKong, 2000; 333-336

Summers RL. Physiology and biophysics of the 100 m sprint. News Physiol Sci, 1997; 12: 131-136

Van Ingen Schenau G J. De Koning JJ, De Groot D. Optimisation of sprinting performance in running, cycling and speed skating. Sports Med, 1994; 17:259-275

Watts AS, Coleman I, Nevill AM. The changing shape characteristics associated with success in world-class sprinters. J Sports Sci, 2011; 30 (11):1085-95

Weyand PG, Sternlight BD, Bellizzi JM, Wright S. Faster top running speeds are achieved with greater ground forces not more rapid leg movements. J Appl Physiol, 2000; 89: 1991-1999

Wood G. Biomechanical limitations to sprint running. [w:] van Gheluwe J, Atha B.(red) Medicine and Sport Science, Basel: Karger, 1987; 58-71

\section{Corresponding author:}

Krzysztof Maćkała

University School of Physical Education in Wroclaw, Department of Track and Field,

ul. Paderewskiego 35, 51-612 Wrocław, Poland

Phone: +48713473140

Fax: +48713473149

E-mail: krzysztof.mackala@awf.wroc.pl 